\title{
A Wavelet-Based ECG Delineation and Automated Diagnosis of Myocardial Infarction in PTB Database
}

\author{
Rachid HADDADI *, Elhassane ABDELMOUNIM*, Mustapha EL HANINE*, \\ Abdelaziz BELAGUID** \\ haddadirachid@hotmail.com, hassan.abdelmounim@hotmail.fr,melhanine@yahoo.fr \\ * Laboratory of Systems Analysis and Information Processing, Faculty of Sciences and Technics, Hassan \\ first University, Settat, Morocco. \\ ** Laboratory of physiology, Mohammed-V University Rabat, Morocco
}

\begin{abstract}
In this work, we present an ECG delineation and the automated diagnosis of coronary artery disease in the electrocardiogram (ECG). In preprocessing stage, the baseline wander (BLW) and $60 \mathrm{~Hz}$ power line interference (PLI) were removed using discrete wavelet transform (DWT). The QRS detection is carried out using Daubechies (Db4) DWT. Feature extraction and classification is done using a convolutional neural network $(\mathrm{CNN})$ containing three convolutional layers, three max-pooling layers, and three fully connected layers. The standard 12 lead ECG signals of 50 healthy subjects and 50 myocardial infarction subjects (MI) of one minute are obtained from the PhysikalischTechnische Bundesanstalt (PTB) database. We achieved an accuracy of $94.83 \%$. sensitivity of $94.75 \%$, and specificity of $94.93 \%$ on PTB database.
\end{abstract}

Keywords: Electrocardiogram, DWT, QRS complex, Convolutional neural network.

\section{Introduction}

Cardiovascular disease is the leading cause of death in the world. According to the World Health Organization (WHO), more than 17.7 million people die each year due to cardiovascular disease, representing $31 \%$ of total global mortality [1]. This number is in permanent growth. The main risk factors are obesity, tobacco, high blood pressure, diabetes and genetic factors. Ischemic heart disease happens when coronary arteries get narrower and reduce the blood flow to the heart. This is also called coronary heart disease (CHD). This can ultimately lead to the heart attack [2]. The electrocardiogram (ECG) is the most used cardiological examination at hospitals and cardiology offices to diagnose heart disease. It is easy to set up, painless and inexpensive.

As illustrated in Figure 1, an ECG is a series of waves and deflections recording the heart's electrical activity from a certain view. Each view called a lead, monitor voltage changes between electrodes placed in different positions on the body. Leads I, II, and III are bipolar leads. Leads aVR, aVL, aVF, V1 through V6 are unipolar leads. An ECG tracing looks different in each lead because the recorded angle of electrical activity changes with each lead [3]. The ECG signal is characterized by a recurrent wave sequence of $\mathrm{P}, \mathrm{QRS}$, and T-wave associated with each beat. Each wave of the ECG represents a phase of heart functioning. Each deformation detected on the duration and the forms of these waves can be considered as an indicator of cardiac abnormalities. 


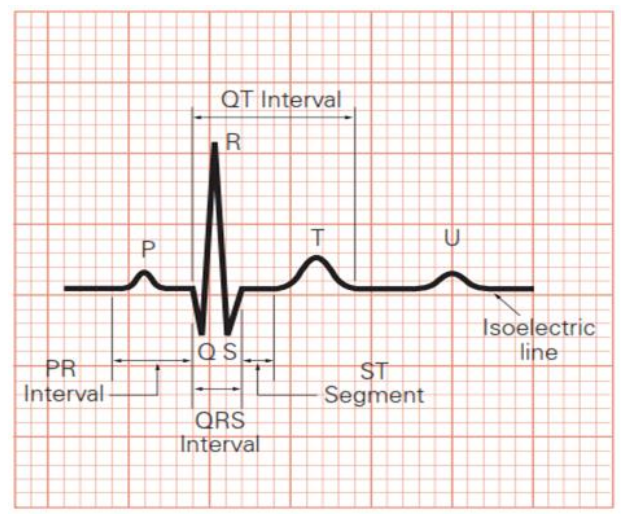

Fig.1. ECG segments and time intervals

Ischemia and Myocardial Infarction (MI) are among the most serious cardiac abnormalities. These anomalies appear on the ECG signal as ST segment or T wave changes. The ST segment elevation and depression indicate the myocardial ischemia. ST segment elevation is measured from the isoelectric line level (the flat part of the ECG between the P wave and the QRS complex) to the J point. The J point is the "junction" point between the end of QRS complex and the onset of ST segment.

There have been several methods dealing with automated characterization of Coronary Artery Disease (CAD). Sun et al. [4] proposed myocardial infarction detection, in 12 ECG leads, using multiple instance learning technique. Authors uses Support vector machine (SVM), NN and k-nearest neighbor (KNN) classifiers. They achieved MI detection sensitivity $92.3 \%$ and specificity $88.1 \%$. Harldsson et al [5] used Hermite expansions with NN technique to classify MI in 12 leads ECG. They achieved ROC area 83.4\%. Lahiri et al. [6] proposed MI detection in 12 leads ECG using Phase space fractal dimension of ECG and Artificial neural network. They obtained sensitivity $96,55 \%$ and specificity $95,24 \%$.

Sharma et al. [7] proposed MI classification in 12-lead ECG using wavelet transform and SVM. Over 549 records of PTB databases, they achieved MI detection sensitivity 93\%, specificity $99 \%$ and accuracy 96\%. In Ref. [8], Arif et al. proposed KNN classifier to detect MI in 12-lead ECG. They used 3200 Normal beats and 16,960 MI and obtained sensitivity $99.97 \%$ and specificity 99.9\%. Zheng et al. [9] used Naïve Bayes, SVM and Random Forest classifiers to detect the presence of old myocardial infarction in body surface potential maps. They achieved MI detection accuracy $81.9 \%$ with Naïve Bayes, $82.8 \%$ with SVM and $84.5 \%$ with Random Forest classifier. In Ref. [10] Acharya et al. proposed KNN classifier for automated detection and localization of myocardial infarction in 12-lead ECG. They used 125,652 normal beats and 485,753 MI from PTBDB and obtained sensitivity $99.45 \%$, specificity $96.27 \%$ and accuracy $98.80 \%$. Acharya et al. [11] used KNN and decision tree (DT) classifiers to detect (CAD) in 12-leads ECG. With Bispectrum-KNN, they achieved accuracy $98.2 \%$ sensitivity $94.8 \%$, and specificity $99.3 \%$. Pyakillya et al. [12] proposed Deep Learning algorithm for ECG Arrhythmia Classification using convolutional neural network (CNN). They used 1-dimentional (1D) CNN architecture comprising 7 convolutional layers with filter width 5 and 128 neurons + max-pooling and dropout after every layer + Global Average Pooling + 3 FCN layers with $(256 / 128 / 64)$ neurons + dropout after every layer + softmax layer with 4 outputs (4 classes: normal sinus rhythm, AF, other rhythm and noisy). The best resulted accuracy on validation data is about $86 \%$. Andrew Ng's scientific group (Pranav Rajpurkar, Awni Y. Hannun, 
Masoumeh Haghpanahi, Codie Bourn) [13] proposed 34-layer CNN architecture for Arrhythmia Detection. They exceed the average cardiologist performance in both recall (sensitivity) and precision (positive predictive value). Liu et al. [14] proposed Real-time Multilead CNN (MLCNN) for MI detection. To make the ML-CNN suitable for multilead ECG processing, they use 1-D kernels shared among the different leads to generate local optimal features. they achieved sensitivity $95.40 \%$, specificity $97.37 \%$ and accuracy $96.00 \%$. T. Reasat et al. [15] used shallow convolutional neural networks to detect Inferior Myocardial Infarction (IMI) in 3 leads ECG signal; lead II, III and aVF. The performance of the model is evaluated on IMI and healthy signals obtained from PTB database. They achieved an accuracy, sensitivity and specificity of $84.54 \%, 85.33 \%$ and $84.09 \%$, respectively.

In this work, we propose an deep convolutional neural networks $(\mathrm{CNN})$ in the classification of two ECG classes; normal and IMI.

\section{Methodology}

\subsection{Data collection}

ECG signals used in this study are taken from the Physikalisch-Technische Bundesanstalt (PTB) database [16]. The database contains 549 records from 290 subjects (aged 17 to 87, mean 57.2; 209 men, mean age 55.5, and 81 women, mean age 61.6; ages were not recorded for 1 female and 14 male subjects).

Each subject is represented by one to five records. There are no subjects numbered 124, 132, 134, or 161. Each record includes 15 leads: the conventional 12 leads (I, II, III, aVR, aVL, aVF, V1, V2, V3, V4, V5, V6) together with the 3 Frank lead ECGs (Vx, Vy, Vz). Each signal is digitized at 1000 samples per second, with 16-bit resolution [17].

In this work, we used ECG signal from lead II, III and aVF and focused on inferior myocardial infarction (IMI) and healthy signals.

\subsection{Preprocessing}

In the preprocessing stage, the Baseline wander (BW) and $60 \mathrm{~Hz}$ power line interference (PLI) must be removed. The removal of this disturbance is an important step in ECG signal analysis, to produce a stable signal for subsequent automatic processing [18].

The method that we propose to eliminate the baseline is based on DWT decomposition up to level 8, which generates a set of approximation coefficients(C8), and eight sets of detail coefficients $(\mathrm{d} 1, \ldots, \mathrm{d} 8)$. By cancellation of approximations, the filtered signal is recovered from the details only [19]. Some results in the removal of baseline wanders is shown in Figure 2. For removal of $60 \mathrm{~Hz}$ PLI interference, we propose to add a sine wave of $60 \mathrm{~Hz}$ frequency to the ECG test signals, to exemplify the original ECG signal as contaminated with PLI. The signal is recovered from all coefficients except details $\mathrm{D}(7,58), \mathrm{D}(7,59)$ and $\mathrm{D}(7,60)$. This cancels all frequencies in the band $60 \pm 1.5 \mathrm{~Hz}$ [20]. The results in removal of $60 \mathrm{~Hz}$ PLI interference are shown in Figure. 3. [20]. 


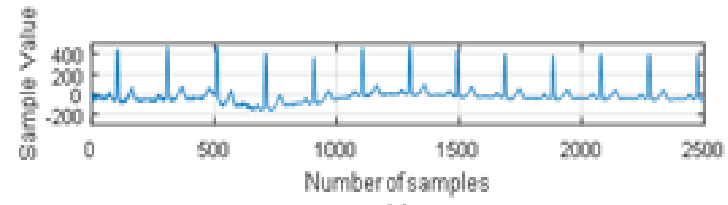

(a)

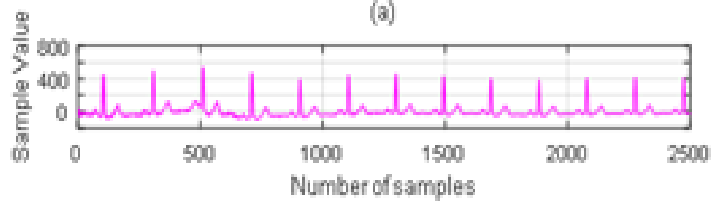

(b)

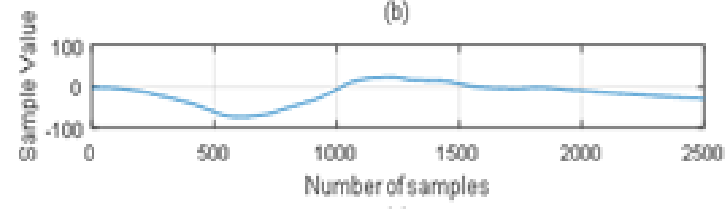

(c)

Fig. 2. Results for removal of baseline wanders: (a) noised ECG: (b) Baseline free ECG; (c) Removed baseline

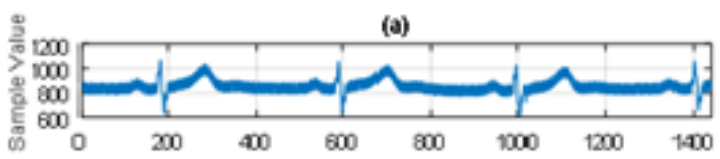

(b)

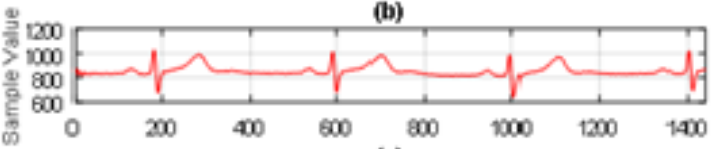

(c)

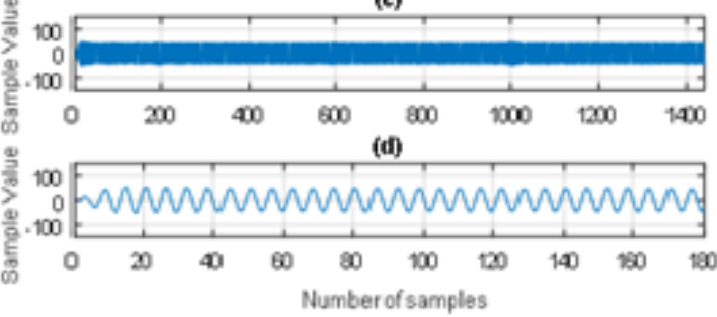

Fig. 3. Results in removal of PLI interference: (a) noised ECG; (b) Filtered ECG; (c) $60 \mathrm{~Hz}$ power line noise; (d) $60 \mathrm{~Hz}$ noise zoomed in $0.5 \mathrm{~s}$.

\section{Detection of QRS complexes}

The QRS complex reflects the electrical activity within the heart during the ventricular contraction, the time of its occurrence, as well as its shape, provide much information about the current state of the heart. The QRS detection is the most important step for almost all ECG analysis algorithms. Pan and Willis J.Tompkins were the first to develop a real-time QRS detection algorithm that uses an automatically adjustable threshold [21]. In this work, the decomposition of the ECG signal is made using Daubechies (Db4) DWT up to level 8. To choose the best coefficient, we compare the correlation coefficient between all the detail coefficients individually with the original ECG signal. The coefficient d4 [8.125Hz-16.25Hz] has the highest cross-correlation compared to other coefficients. Therefore, in the time domain, d4 is highly correlated with the original signal. The ECG signal is reconstructed with the coefficient d4. To detect the R Peaks, we use a hard thresholding method [19]. Figure 4 shows the original ECG signal with R peaks localization. The algorithm is also evaluated, in 12 leads PTB database, using Two performance measures : sensitivity $(\mathrm{Se})$ and Positive predictivity $(+\mathrm{P})$ defined respectively as:

$$
\begin{gathered}
S e=\frac{T P}{T P+F N} \\
+P=\frac{T P}{T P+F P}
\end{gathered}
$$




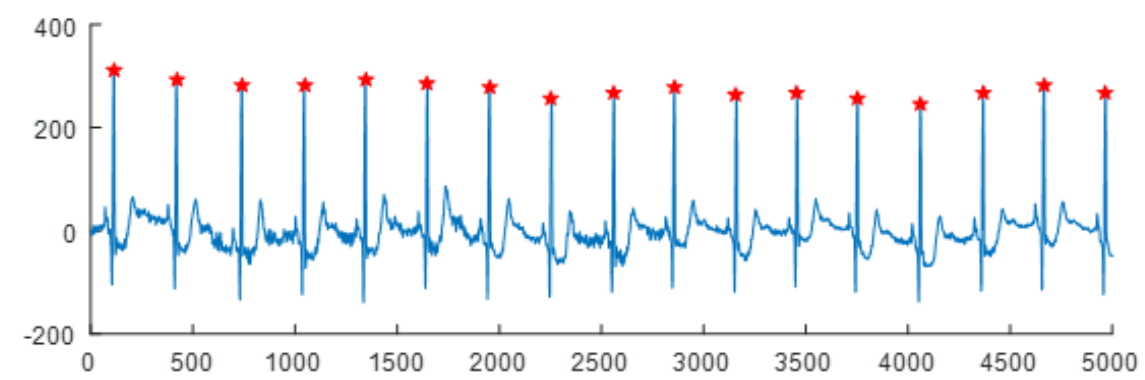

Fig. 4. Original ECG signal with R peak detection.

Where TP means a true positive, FN means a false negative and FP denotes a false positive detection.

Table 1 illustrates the one-minute results for three patients from PTB database:

Table 1. Results for 1 minute $\mathrm{R}$ peaks detection from PTBDB

\begin{tabular}{|c|c|c|c|c|c|c|}
\hline Patient & Records & $\mathrm{TP}$ & FN & FP & $\mathrm{Se}(\%)$ & $+\mathrm{P}(\%)$ \\
\hline \multirow{3}{*}{ PTB P.013 } & s00451rem & 980 & 5 & 4 & 99.49 & 99.59 \\
\hline & s0051lrem & 957 & 5 & 5 & 99.48 & 99.48 \\
\hline & s0072lrem & 1032 & 5 & 5 & 99.52 & 99.52 \\
\hline \multirow{3}{*}{ РТВ P.019 } & s00581rem & 1032 & 4 & 7 & 99.61 & 99.33 \\
\hline & s00701rem & 986 & 4 & 5 & 99.60 & 99.50 \\
\hline & s0077lrem & 847 & 5 & 6 & 99.41 & 99.30 \\
\hline \multirow{3}{*}{ PTB P.021 } & s0065lrem & 852 & 5 & 5 & 99.42 & 99.42 \\
\hline & s00731rem & 1041 & 5 & 3 & 99.52 & 99.71 \\
\hline & s00971rem & 1064 & 4 & 5 & 99.63 & 99.53 \\
\hline \multirow{2}{*}{ РTB P.117 } & s02911rem & 780 & 5 & 6 & 99.36 & 99.24 \\
\hline & s02921rem & 790 & 4 & 5 & 99.50 & 99.37 \\
\hline \multicolumn{2}{|c|}{ Total } & 10361 & 51 & 56 & 99.51 & 99.46 \\
\hline
\end{tabular}

\section{CNN based Classifier}

Convolutional neural networks (CNN) have emerged from the study of the visual cortex of the brain, and they have been used in image recognition since the 1980s "the neocognitron" [22], which has progressively evolved into what we today call the convolutional neural networks. An important step was taken in 1998 by Yann LeCun, Bottou, Yoshua Bengio and Patrick Haffner, who introduced the famous LeNet-5 architecture, widely used to recognize handwritten check numbers. 


\subsection{Pre-processing}

Each ECG beat consists of a maximum of 670 samples (fs $=1 \mathrm{KHz}$ ); considering the maximum values of the PR and QT intervals (PRmax + QTmax $=200 \mathrm{~ms}+470 \mathrm{~ms}=670 \mathrm{~ms}$ ). This value will be the size of the input vector $(m=670)$. To make sure that the $\mathrm{P}, \mathrm{QRS}$ and $\mathrm{T}$ waves are included, we use the positions of the $\mathrm{R}$ peaks.

To solve the problem of amplitude scaling and eliminate the shift effect before introducing the ECG segments into the one-dimensional CNN, each segment of the ECG signals is normalized with the Z-score normalization method.

\subsection{The architecture}

In this work, we use a CNN architecture comprising 3 convolutional layers, 3 subsampling layers (Max pooling) and 3 fully connected layers. Each convolutional layer is followed by LeakyRelu [23] activation function to recover the non linearity into the data [24]. The output layer of the last fully connected network is a Softmax layer (layer 9) with a two-dimensional "vector" output since this is a two-class problem (normal and IMI). Figure 5 illustrates the overall structure of our proposed CNN. Table 2 summarizes the details of this CNN structure.

The number of neurons at the output of each channel was evaluated using this formula:

$$
o=\left\lfloor\frac{n+2 p-m}{s}\right\rfloor+1
$$

where $\mathrm{n}$ the length of input vector, $\mathrm{m}$ the Kernel size, $\mathrm{p}$ the padding $(\mathrm{p}=0)$ and $\mathrm{s}$ the Stride.

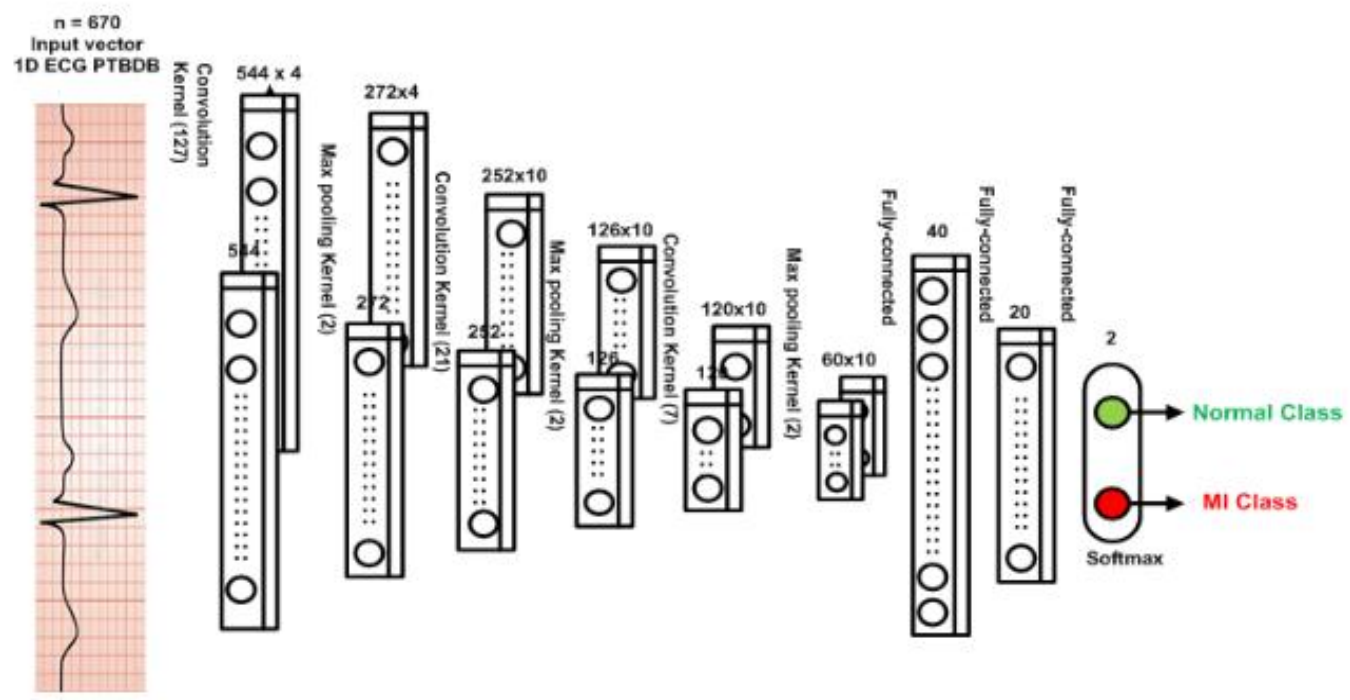

Fig. 5. The proposed CNN architecture 
Table 2. Details of the CNN structure

\begin{tabular}{cccc}
\hline \multicolumn{3}{c}{ Input vector size (670) } \\
\hline Layers & $\begin{array}{c}\text { Kernel } \\
\text { size }\end{array}$ & stride & $\begin{array}{c}\text { Number of neurons } \\
\text { for each channel }\end{array}$ \\
\hline (1) Convolution & 127 & 1 & 544 \\
(2) Max pooling & 2 & 2 & 272 \\
(3) Convolution & 21 & 1 & 252 \\
(4) Max pooling & 2 & 2 & 126 \\
(5) Convolution & 7 & 1 & 120 \\
(6) Max pooling & 2 & 2 & 60 \\
(7) Fully connected & & & 40 \\
(8) Fully connected & & & 20 \\
(9) Fully connected & & & 2 \\
\hline
\end{tabular}

\subsection{Experiments and Results}

In this study, we use a backpropagation, based on stochastic gradient descent (SGD), to adjust the momentum, regularization and learning rate. The best performances were obtained by adjusting the Momentum at $5 \times 10-3$, the learning rate 0.6 , and the Regularization at 0,1 . We performed several epochs of training and testing. We validates the CNN model for each epoch. The classification error is stabilized and has converged after 45 epochs.

To validate our model, we used 10-fold cross validation. The performances (Accuracy, sensitivity, and specificity) are evaluated at each iteration. The overall performance is the average performance recorded in the 10 iterations. To make a better comparison between various performance indices, the confusion matrix presented in Table 3 is used. Overall performance with optimal settings is shown in Figure 6.

Table 3. Confusion matrix of the CNN classifier

\begin{tabular}{ccc}
\hline & \multicolumn{2}{c}{ Predicted } \\
\cline { 2 - 3 } & Normal & IMI \\
\hline Normal & 1780 & 95 \\
IMI & 1895 & 105 \\
\hline
\end{tabular}

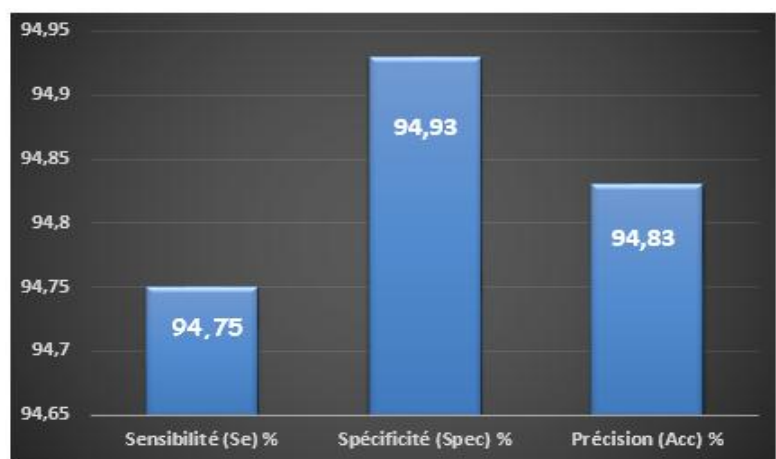

Fig. 6. Results of the CNN classifier 


\subsection{Discussion}

Table 4 shows some techniques used in the literature for the automated classification of myocardial infarction validated on the same database (PTBDB).

Table 4. Comparison of our MI classifiers with other methods in PTB database

\begin{tabular}{cccccc}
\hline \multirow{2}{*}{ Author, Year } & \multirow{2}{*}{ Ref } & \multirow{2}{*}{ Classifier } & \multicolumn{3}{c}{ Performance } \\
\cline { 4 - 6 } & & & $\%$ Se & $\%$ Spec & $\%$ Acc \\
\hline Sun et al., 2012 & {$[25]$} & SVM & 92,60 & 82,4 & - \\
Safdarian et al., 2014 & {$[26]$} & Naïve Bayes & - & - & 94,74 \\
Lu et al. 2000 & {$[27]$} & Fuzzy ANN & 84,60 & 90 & - \\
Liu et al., 2017 & {$[28]$} & CNN & 95,40 & 97,37 & 96 \\
Our work & & CNN & 94,75 & 94,93 & 94,83 \\
\hline
\end{tabular}

The performance of our system is not the best, but it exceeds several works cited in the table above. Our precision is close to that of Liu et al. [28] obtained with a CNN network. This last work uses a larger data size than ours, with 122 training records and 125 test records, for a total of 34769 12-lead beats. In our study, we used only 100 ECG recordings of one minute, on three leads DII, DIII and aVF; commonly used derivations for the diagnosis of inferior infarction. This choice is justified by a hardware limitation since we use the CPU version of Keras-tensorflow. To reduce the calculation time and increase the data size, we have to use the GPU version.

\section{Conclusion and future work}

In this paper, we present an algorithm of automatic detection of inferior myocardial infarction based on convolutional neural network (CNN), using leads II, III and aVF from PTB database. In the preprocessing stage, discrete wavelet transform (DWT) was used to remove the baseline wander and power line interference. DWT was also used for QRS detection. The filtered signals are normalized and sent directly to the CNN network. We achieved an average sensitivity, specificity and accuracy of $94,75 \%, 94,93 \%$ and $94,83 \%$ respectively.

In this study, we used a binary classification that does not provide information about the location of myocardial infarction. Indeed, there are 10 types of infarction in the PTB database, classified according to the location in the myocardium. In the future, our CNN classifier could be extended to an 11-class classifier to be able to recognize the different types of infarction.

\section{References}

[1] World Health Organization (WHO), "Cardiovascular Diseases," (2017)

[2] http://www.heart.org/HEARTORG/Conditions/HeartAttack/PreventionTreatmentofHeartAttack/Sil ent-Ischemia-and-Ischemic-Heart-Disease_UCM_434092_Article.jsp\#.WYmRzITyjIU.

[3] Shirley A. Jones, ECG Success Exercises in ECG Interpretation, MS Ed, MHA, EMT-P (2008)

[4] L. Sun, Y. Lu, K. yang, S. Li, "ECG analysis using multiple instance learning for myocardial infarction detection,"IEEE Trans. Biomed. Eng. 9 (12), pp. 3348-3356 (2012) 
[5] H. Haraldsson, L. Edenbrandt, M. Ohlsson, "Detecting acute myocardial infarction in the 12-lead ECG using Hermite expansions and neural networks, "Artif. Intell. Med, vol. 32, pp. 127-136 (2004)

[6] T. Lahiri, U. Kumar, H. Mishra, S. Sarkar, A.D. Roy, "Analysis of ECG signal by chaos principle to help automatic diagnosis of myocardial infarction, ” J. Scient. Industr. Res, vol.68, pp. 866-870 (2009)

[7] L.N. Sharma, R.K. Tripathy, S. Dandapat, "Multiscale energy and eigenspace approach to detection and localization of myocardial infarction, " IEEE Trans. Biomed. Eng., vol. 62 (7), pp. 1827-1837 (2015)

[8] M. Arif, I.A. Malagore, F.A. Afsar, "Detection and localization of myocardial infarction using knearest neighbor classifier, ” J. Med. Syst., vol. 36 (1), pp. 279-289 (2012)

[9] H. Zheng, H. Wang, C.D. Nugent, D.D. Finlay, "Supervised classification models to detect the presence of old myocardial infarction in body surface potential maps, "In: Proc. of Computers in Cardiology, pp. 265-268 (2006)

[10] R. Acharya et al." Automated detection and localization of myocardial infarction using electrocardiogram: a comparative study of different leads, " Knowledge-Based Syst., vol. 99, pp. 146-156 (2016)

[11] U.R. Acharya et al. "Application of higher-order spectra for the characterization of coronary artery disease using electrocardiogram signals, " Biomed. Signal Process. Control, vol. 31, pp. 31-34 (2017)

[12] B. Pyakillya, N. Kazachenko, and N. Mikhailovsky, "Deep Learning for ECG Classification," IOP Conf. Series: Journal of Physics: Conference Series, vol. 913, no. 1, pp. 1-5 (2017)

[13] Rajpurkar P, Hannun Awni Y, Haghpanahi M, Bourn C and Ng Y. Ng, “A 2017 Cardiologist-Level Arrhythmia Detection with Convolutional Neural Networks," (Preprint arXiv:1707.01836v1).

[14] Wenhan Liu et al., "Real-time Multilead Convolutional Neural Network for Myocardial Infarction Detection," IEEE Journal of Biomedical and Health Informatics, vol. 99, pp. 1-12 (2017)

[15] T. Reasat, C. Shahnaz, "Detection of Inferior Myocardial Infarction using Shallow Convolutional Neural Networks", In: Proc. of 2017 IEEE Region 10 Humanitarian Technology Conference (R10HTC), 21 - 23 Dec 2017, Dhaka, Bangladesh, pp. 718-721 (2017)

[16] Goldberger et al., "PhysioBank, PhysioToolkit, and PhysioNet: Components of a New Research Resource for Complex Physiologic Signals", Circulation, 101(23): e215-e220

[17] https://physionet.org/physiobank/database/ptbdb

[18] Bailey et al. "Recommendations for the Standardization and Specifications in Automated Electrocardiography: Bandwidth and Signal Processing", Circulation, vol.81, 730-739 (1990)

[19] R.Haddadi, E.Abdelmounim, M.El Hanine, and A.Belaguid, "Discrete Wavelet Transform Based Algorithm for Recognition of QRS Complexes," World of Computer Science and Information Technology Journal (WCSIT), Vol. 4, No.9, pp. 127-132, (2014)

[20] R. Haddadi, E. Abdelmounim, M. El Hanine, and A. Belaguid, "ST Segment Analysis Using Wavelet Transform," IJCSNS International Journal of Computer Science and Network Security, vol. 17, no. 9, pp. 102-107, September (2017)

[21] J. Pan, J. Tompkins, “A Real-Time QRS Detection Algorithm, ” IEEE Trans. Biomed. Eng. BME, vol. 32 (3), pp. 230-236 (1985)

[22] K. Fukushima, "Neocognitron: A Self-organizing Neural Network Model for a Mechanism of Pattern Recognition Unaffected," Biol. Cybernetics, vol. 36, no. 4, pp. 193-202, (1986).

[23] He.K. Zhang, X. Ren, S. Sun, Delving deep into rectifiers: surpassing human-level performance on image net classification, 1026-1034, (2015)

[24] Y. LeCun, Y. Bengio, G. Hinton, Deep learning, Nature 521 436-444(2015)

[25] Li Sun, Yanping Lu, Kaitao Yang, and Shaozi Li, "ECG Analysis Using Multiple Instance Learning for Myocardial Infarction Detection", IEEE Transactions on Biomedical Engineering, vol. 59, no. 12, pp. 3348 - 3356, (2012) 
[26] N. Safdarian, N. J. Dabanlo, and G. Attarodi, "A New Pattern Recognition Method for Detection and Localization of Myocardial Infarction Using T-Wave Integral and Total Integral as Extracted Features from One Cycle of ECG Signal", Journal of Biomedical Science and Engineering, vol. 7, no. 10, pp. 818-824 ( 2014)

[27] H.L. Lu, K. Ong, and P. Chia, "An automated ECG classification system based on a neuro-fuzzy system", Computers in Cardiology 2000, vol. 27, pp. 387-390 (2000)

[28] Wenhan Liu et al., "Real-time Multilead Convolutional Neural Network for Myocardial Infarction Detection", IEEE Journal of Biomedical and Health Informatics, vol. 99, pp. 1-12 (2017) 